\title{
Elproceso de formación deportiva en la iniciación alos deportes colectivos fundamentado en las características del deportista experto The process of training from the team sport initiation based on the expert characteristics
}

\author{
José Luis Arias Estero \\ *Universidad Católica San Antonio de Murcia (España)
}

\begin{abstract}
Resumen: La nueva aproximación de formación de expertos ha modificado la antigua visión del proceso de detección de talentos. Dicha perspectiva consiste en evaluar a los deportistas expertos, para analizar su proceso de formación y establecer las variables clave que los diferencia. A consecuencia de este cambio en la comprensión del talento, lo que se pretende con este trabajo es analizar las características que indica la bibliografia sobre los expertos para orientar el proceso de formación deportiva en la fase de iniciación a los deportes colectivos. La iniciación deportiva debe encaminarse al desarrollo de patrones sociales, psicológicos, cognitivos y motrices. El tiempo necesario para aprender, la cantidad de tiempo empleado a un nivel apropiado de dificultad y la cantidad o variabilidad de las experiencias, son variables que marcan el éxito en la enseñanza. Desde un punto de vista global, los aspectos que caracterizan al deportista experto, son los factores genéticos, los de entrenamiento y los aspectos psico-sociales. Sin embargo, la compleja relación entre las distintas variables que influyen en el proceso dificultan la definición del experto. A partir de esta realidad, se elabora una propuesta basada en ocho aspectos fundamentales para el entrenamiento y la formación deportiva integral desde la iniciación a los deportes colectivos.
\end{abstract}

Palabras clave: Experto, iniciación deportiva, proceso de enseñanza, práctica.

Abstract: The new approach of expert's formation has modified the old vision of the talents detection process. This perspective consists of evaluating the expertise, to analyze their formation process and to establish the key that difference them. The purpose of this work is to analyze the expertise characteristics that the bibliography indicates to orient the process of training from the team sport initiation. The sport initiation must be directed to the development of social, psychological, cognitive and motor patterns. The time necessary to learn, the amount of time used at an appropriate level of difficulty and the amount or variability of the experiences, are variables that mark the success in sport formation. From a global point of view, the aspects that characterize the expertise are the genetic, the training and the psycho-social factors. However, the complex relationships are considerably affected by any uncertainty in the behaviour of the variables. From this reality, a training proposition is proposed. The training proposition is based on eight fundamental aspects to the training and to the integral sport formation from the team sport initiation.

Key words: Expertise, sport initiation, teaching-learning process, practice.

\section{Introducción}

Desde un enfoque deportivo se entiende que la primera etapa del desarrollo formativo del jugador es la iniciación deportiva. Ésta debe considerarse como un periodo de adquisición de habilidades, de socialización, de toma de contacto y de enseñanza-aprendizaje (Giménez \& Sáenz-López, 2000), sujeta al cumplimiento de unos principios sociales, pedagógicos, psicológicos, biológicos y motrices, que garanticen el desarrollo del jugador (Blázquez, 1995; Pierón, 1989).

Con motivo de asegurar el desarrollo del jugador, en la etapa de iniciación deportiva se abre una gran área de investigación centrada en la búsqueda de talentos. El proceso de búsqueda de talentos se ha asociado, tradicionalmente en el campo de la actividad fisica y del deporte, a la posesión de aptitudes, características o capacidades especiales para poder alcanzar altos resultados deportivos. En este sentido, dichas características especiales han sido evaluadas en términos de medidas antropométricas, cualidades físicas, cualidades motrices, capacidades de aprendizaje y predisposición para el aprendizaje (Solanellas, 1999). Sin embargo, en la actualidad, este término se ha visto remplazado por el de experto.

El término experto, como indican Ruiz \& Sánchez (1997), hace referencia a alguien experimentado, es decir, a alguien enseñado durante un tiempo bajo un plan de trabajo y supervisión, que además, muestra una voluntad y aptitud de mejora y perfeccionamiento. Por lo que se puede decir, que la visión antigua del talento ha sido reemplazada por la realidad del experto. Esta nueva visión se basa, principalmente, en la importancia que se le supone al proceso de entrenamiento y en concreto a la cantidad de práctica y experiencias acumuladas, en contraposición de la mayor trascendencia que tienen las características genéticas bajo la concepción de talento.

Fecha recepción: 04-10-07 - Fecha envío revisores: 08-10-07 - Fecha de aceptación: 05-11-07 Correspondencia: José Luis Arias Estereo

$\mathrm{C} /$ La Labor 1

30520 Jumilla - Murcia (España)

E-mail: jlae84@hotmail.com
La nueva aproximación de formación de expertos ha modificado la antigua visión del proceso de detección de talentos. Se ha observado cómo el proceso de detección clásico, consistente en proponer a los deportistas considerados expertos como referencias para realizar valoraciones y establecer pruebas adecuadas, está siendo sustituido por una nueva perspectiva. Dicha perspectiva consiste en evaluar a los deportistas considerados expertos, para analizar su proceso de formación y así poder encontrar las variables clave que marcan las diferencias entre los deportistas considerados expertos y el resto. A la vez este tipo de análisis se puede hacer desde dos vertientes: a) examinando la formación de los referidos expertos consolidados y/o b) comparando a los expertos con los no expertos de la misma edad (Lorenzo, 2003). Aunque en la actualidad, están apareciendo diferentes estudios en los que una o diversas variables son comparadas en muestras de deportistas de diferentes niveles de destreza (Ruiz, Sánchez, Durán \& Jiménez, 2006).

A consecuencia de este cambio en la comprensión del talento y fruto de las modalidades de análisis que surgen, lo que se pretende con este trabajo de revisión es analizar las características que indica la bibliografia sobre los expertos para orientar el proceso de formación deportiva en la fase de iniciación de los deportes colectivos. Para la realización de este trabajo no se ha seguido ningún proceso restrictivo en la búsqueda y elección bibliográfica. Por este motivo a partir de términos considerados clave se procedió a la búsqueda bibliográfica en diferentes bases de datos y en revistas con versión en papel y electrónica.

\section{La formación en la iniciación deportiva}

La iniciación deportiva, como proceso general por el que cualquier individuo se forma en uno o varios deportes, se fundamenta en la adquisición de los patrones cognitivo-motrices básicos sobre los que asentar el posterior aprendizaje de la toma de decisión y las habilidades motrices específicas de un deporte (Hernández, et al., 2000). El proceso de iniciación debe encaminarse al desarrollo de patrones sociales, psicológicos, cognitivos y motrices. En dicho desarrollo, la práctica deportiva desempeña una labor esencial, en tanto que contribuye al progreso del ser humano (Committee on Sports Medicine and Fitness 
\& Committee on School Health, 2001; Ginsburg, Committee on Communications \& Committee on Psychosocial Aspects of Child and Family Health, 2007). Para contribuir a la evolución del ser humano, la iniciación deportiva debe basarse en la reflexión y la comprensión, buscando una mejora integral del individuo a partir de la creación de hábitos motores. No se debe considerar al niño como autómata, sino que se debe partir de la globalidad del deporte en cuestión para desarrollar sus capacidades (Del Villar \& Iglesias, 2003).

La iniciación deportiva, con objeto de desarrollar las capacidades de los niños, debe responder a sus necesidades, intereses y posibilidades. No se deben exigir resultados inmediatos (Silva, Fernandes \& Celani, 2001), asumiendo el fallo como parte del aprendizaje. No hay que olvidar que en esta etapa, por su carácter de toma de contacto con el deporte, los contenidos hay que presentarlos de forma atractiva y motivante. Los niños tienen que divertirse con lo que hacen, puesto que es una característica propia de la edad. Partiendo de estas premisas y debido a que es en esta etapa cuando se constituirá la base sobre la que se debe asentar la formación posterior como persona y deportista, desde la iniciación deportiva resulta necesaria la adquisición de un gran número de experiencias (Piñar \& Cárdenas, 2004).

La construcción de nuevos aprendizajes depende, muy directamente, del nivel de conocimiento previo y éste de las experiencias previas acumuladas a través de la práctica. A pesar de esto, las habilidades cognitivo-motrices no se desarrollan más rápido sólo por enseñarlas a más temprana edad (Committee on Sports Medicine and Fitness \& Committee on School Health, 2001). El tiempo necesario para aprender (Silverman, Devillier \& Ramirez, 1991), la cantidad de tiempo empleado a un nivel apropiado de dificultad (Lee, 1996) y la cantidad o variabilidad de las experiencias (Schmidt, 1975), son tres variables que marcan el éxito en la enseñanza. Por lo que el hecho de practicar más, de forma adaptada a los niños, facilita el desarrollo formativo, en tanto que a mayorparticipaciónmayor construcción de esquemas(Côté, Macdonald, Baker \&Abernethy, 2006), que serán mejorados mediante su variabilidad. Lo cual, aunque pueda parecer una idea básica, no refleja la realidad de la mayoría de planteamientos de iniciación deportiva al que son sometidos los individuos.

\section{El tiempo de práctica}

El tiempo de práctica es fundamental para garantizar una formación adecuada. Un mayor tiempo de participación permite mayores posibilidades de formación deportiva. Además, si la práctica se produce en contacto con el móvil, ya sea un balón u otro tipo de móvil que conlleve el mismo fin, dicha práctica será más significativa. Por lo que el tiempo de práctica se considera un factor que debe ser controlado por el entrenador para proporcionar las mismas opciones de desarrollo a todos los jugadores (Blázquez, 1995; Cárdenas, 2003; Damas \& Julian, 2002).

\section{La adaptación de la práctica}

La adaptación de la práctica es una estrategia facilitadora del aprendizaje y del desarrollo en la iniciación (Del Villar, Julián, Iglesias \& Fuentes, 2003). La adecuación de la práctica a los niños persigue que el planteamiento de enseñanza esté acorde a las necesidades e intereses propios de la etapa (Chalip \& Green, 1998; Dyson, Griffin \& Hastie, 2004), con el fin de que se aumente la participación, las experiencias positivas hacia el deporte y el nivel de destrezas (Chalip \& Green, 1998). De acuerdo con Ginsburg, et al. (2007), cuando la práctica se hace a la medida del niño, a éste se le permite evolucionar a su ritmo, descubrir sus intereses y disfrutar con lo que hace, consiguiendo desarrollar las destrezas cognitivo-motrices.

Para lograrlo se incide sobre tres aspectos: a) la adaptación para obtener éxito inicial con facilidad (Dyson, et al., 2004), para que los niños se sientan capaces y se cree adherencia hacia la práctica; b) la adaptación de los contenidos, posibilitando un nivel de dificultad adecuado que provoque el desarrollo (Boyce, Coker \& Bunker, 2006); y c) la adaptación de los equipamientos, debido a que el equipamiento adulto no es el adecuado al niño, puesto que estos no han alcanzado el nivel de desarrollo de los primeros (Regimbal, Deller \& Plimpton, 1992).

\section{La variabilidad de la práctica}

La variación de las condiciones de práctica en iniciación es un recurso (Williams \& Hodges, 2005). La práctica se debe variar después de superar la fase de los programas motores rudimentarios (Boyce, et al., 2006). Esto es, la adquisición de una base inicial de habilidades sobre la que, posteriormente, introducir variaciones que fortalezcan y asienten las destrezas iniciales. Esta práctica variada se retiene principalmente a largo plazo y permite una mejor transferencia (Green \& Magill, 1995; Landin \& Herbert, 1997).

Mientras, que la práctica variada requiere un aprendizaje lento y complejo, la práctica no variada permite una mejor adquisición de forma más rápida. Por lo que se estipula que la práctica no variada es más adecuada para la obtención más rápida de rendimiento y la variada se relaciona más con el aprendizaje significativo de destrezas (Green \& Magill, 1995; Williams \& Hodges, 2005). No obstante, como señala Hynes (2002) la práctica variada es más apropiada para deportes en los que el entorno no es estable ni predecible, por la continua necesidad de responder ante estímulos cambiantes.

La variabilidad de experiencias cognitivo-motrices es necesaria para la construcción de patrones correctos. Un trabajo variado permite consolidar las estructuras cognitivo-motrices, como consecuencia de aumentar el bagaje de experiencias. La variabilidad permite la adquisición de un gran número de habilidades y éstas a su vez desarrollan un sistema cognitivo-motriz adaptable (Schmidt, 1975).

\section{El experto}

En diferentes trabajos se han examinado una gran cantidad de variables que afectan al desarrollo del rendimiento del experto. Estas variables pueden ser divididas en factores primarios y secundarios (Baker \& Horton, 2004). No obstante, desde un punto de vista global y puesto que resulta inverosímil considerar los diferentes factores influyentes de forma aislada, los aspectos que caracterizan al deportista experto, son: a) los factores genéticos (Baker, Horton, Robertson-Wilson \& Wall, 2003; Rankinen, et al., 2001), aspectos no entrenables o muy poco entrenables, b) los factores de entrenamiento (Helsen, Hodges, Van Winckel \& Starkes, 2000); y c) los aspectos psico-sociales (Hamilton, 2000), que determinan la disposición para el rendimiento.

\section{Factores genéticos}

Según la bibliografía, el aspecto genético es fundamental en el desarrollo del talento deportivo (Baker, et al., 2003; Rankinen, et al., 2002). Implícitos en él están el biotipo, que nos determinará el conjunto de rasgos morfológicos y estructurales que caractericen al deportista; $y$ la edad biológica, considerada por encima de la cronológica y con mayor importancia en los primeros estadios de crecimiento, donde la maduración ósea está en su máximo exponente. No obstante, en la nueva perspectiva del experto los factores genéticos parecen desplazarse a una segunda línea de importancia

Aunque los deportes difieran en las habilidades requeridas para alcanzar el rendimiento, todos ellos coinciden en la importancia de las necesidades fisicas y mentales. Existe literatura significativa que indica la importancia de las características hereditarias paraalcanzarel rendimiento deportivo (Rankinen, etal., 2001, 2002). Será muy importante determinar qué contenidos resultan no entrenables y cuáles muy poco entrenables, para poder orientar el trabajo de entrenamiento de forma adecuada, con el fin de mejorar esos aspectos muy poco entrenables, en el caso que se estipule necesario.

\section{Factores del entrenamiento}

El tiempo de práctica es uno de los aspectos al que más se refiere la 
bibliografía revisada en relación a las variables que caracteriza al deportista experto. Investigaciones rigurosas han analizado el efecto positivo de la práctica prolongada sobre la mejora en el rendimiento(Ericsson, Krampe \& Tesch-Römer, 1993; Ericsson \& Lehmann, 1996). La práctica es necesaria para desarrollar la excelencia deportiva. Está demostrado que grandes cantidades de práctica facilitan el desarrollo del experto deportivo, tanto desde un punto de vista cuantitativo como cualitativo (Baker, Côté, \& Abernethy, 2003; Helsen, et al., 2000). Este efecto se radicaliza bajo el concepto del efecto de la edad relativa. El efecto de la edad relativa determina las diferencias entre sujetos del mismo grupo de edad en función de la fecha de nacimiento. De manera que los sujetos nacidos en los meses iniciales de un determinado año se les suponen mayores posibilidades de convertirse en expertos, que a los nacidos en meses finales de ese mismo año (Baker \& Côté, 2003; Baxter-Jones \& Helms, 1994; Edwards, 1994).

Dentro de la cantidad de tiempo dedicado a practicar, se puede decir que lo que principalmente diferencia al deportista experto del resto son las experiencias que se van a adquirir. $Y$ dentro de las experiencias a adquirir habrá que determinar aquellas que concretamente son importantes por su relevancia en el proceso. Chi, Feltovich \& Glaser (1981), French \& Thomas (1987) y Glaser \& Chi (1988), han demostrado que los expertos muestran un mayor conocimiento procedimental y una habilidad superior para resolver problemas impuestos en actividades. Además, éstos manifiestan un conocimiento más elaborado, estructurado y organizado (Doods, Griffin \& Placek, 2001), que les permite una toma de decisión más rápida (Nielsen \& McPherson, 2001). Lo que indica que el desarrollo de la toma de decisión es uno de los elementos que contribuye al desarrollo del experto (Abernethy, 1990; French \& Thomas, 1987; Rovegno, Nevett \& Babiarz, 2001).

Partiendo de esto, en el proceso de formación deportiva, sería necesario presentar tareas que permitan adquirir experiencias, las cuales aumenten los conocimientos que se poseen, la inteligenciay la creatividad (Baker \& Côté, 2003). Es decir, se deberían evaluar las capacidades previas y a partir de la evaluación proponer tareas que impliquen al individuo en lo que hace, no sólo de forma motriz, sino también cognitivamente. Estas tareas deberían tener una dificultad apropiada individual y grupalmente, de manera que permitiesen al deportista mejorar. Lo cual se convierte en un objetivo difícil cuando se planifican procesos faltos de fundamentos. Ala vez, las tareas deberían demandarun esfuerzo por parte del deportista y la posibilidad de un número adecuado de correctas repeticiones, que la bibliografia suele situar sobre el $70 \%$. Dichas repeticiones posibilitarían el aprendizaje y/o la corrección de los errores que surjan (Baker, et al., 2003), utilizando el feedback con el fin de dar conocimiento de los resultados. Para este fin, es necesario que las tareas tengan objetivos determinados, sobre los que trabajar y que a la vez sirvan de referencia para guiar el proceso (Baker, Côté \& Deakin, 2005).

Según Helsen, et al. (2000), dichos aspectos se cumplen en aquellas actividades que se consideran poco divertidas por los deportistas, pero que son las que les permiten progresar en su desarrollo. Por lo que habrá que intentar motivarlos, mediante la práctica en equipo y atendiendo a sus preferencias y consideraciones siempre y cuando sea posible.

En este sentido, algunas de las características que, a grandes rasgos, pueden orientar el diseño de tareas, para que se cumpla lo comentado en los párrafos anteriores, son:

1. Proponer actividades específicas que permitan adquirir conocimientos específicos (Baker, et al., 2003).

2. Aumentar el tiempo dedicado al entrenamiento intenso y/o intencionado (Helsen, et al., 2000).

3. Que las tareas permitan, de forma implícita, adquirir un alto nivel de preparación psicológica y física (Baker, et al., 2003).

4. Generar situaciones para desarrollar el procesamiento de la información (Baker, et al., 2003), de manera que aumente la capacidad de percepción, especialmente en términos de anticipación y habilidad para captar la información; la capacidad de toma de decisiones, en términos de conocimientos de procedimientos tácticos específicos; y la capacidad para ejecutar los movimientos, especialmente en términos de adaptación al movimiento y automatización de este (Baker, et al., 2003).

5. Que las actividades y las cantidades idóneas de esfuerzo y concentración se modifiquen, permitiendo adquirir más experiencias (Baker, et al., 2003; Helsen, et al., 2000).

Según Baker, et al. (2003), la actividad considerada más útil es la competición. Ésta, a parte de permitir grandes cantidades de esfuerzo físico y cognitivo y a pesar de no estar estrictamente diseñada, constituye un medio idóneo, en el que confluyen todas las necesidades que permitirán la formación que se busca para los deportistas, puesto que la competición exige al sujeto su participación total.

Los responsables de ofrecer a los deportistas los medios esenciales para desarrollar un proceso adaptado serán, principalmente, los entrenadores. Los entrenadores se deben caracterizar por tener una habilidad especial para diseñar programas que favorezcan el desarrollo deportivo de forma óptima (Baker, et al., 2003). Es decir, puesto que los entrenadores son los responsables de la formación del deportista, ellos deben preocuparse de estar formados y actualizados en el área en el que trabajan, conocer las características de sus deportistas y los medios de que se dispone para lograr unos objetivos determinados previamente.

Aunque, la principal característica de los entrenadores en etapas de iniciación es que, deben dominar los fundamentos técnicos básicos y transmitir entusiasmo, no deben dejar de lado aspectos tan importantes como planificar el entrenamiento. Deben prestar atención a todos los detalles, de manera que los objetivos de entrenamiento sean fijados con precisión, para que los deportistas sigan con un ritmo adecuado las exigencias del proceso.Además, deben mantener a los jugadores siempre activos, utilizando tareas adecuadas al nivel de los jugadores, de modo que se haga un mejor uso del tiempo de entrenamiento. Estas tareas deben tener una intensidad similar al de la competición, simulando las condiciones de juego y trabajando bajo presión (Baker, et al., 2003; Piñar \& Cárdenas, 2004; Piñar, 2005).

\section{Aspectos psico-sociales}

La adquisición de la condición de experto está condicionada también por las características psicológicas (Smith \& Christensen, 1995) y sociales (Côté, 1999). Se puede decir que son muchos los factores que influyen $\mathrm{y} / \mathrm{o}$ pueden influir en el proceso de formación deportiva. «Lo que los deportistas piensan de sí mismos y lo que los demás piensan de ellos puede llegar a generar un áurea que les haga invencibles» (Hamilton, 2000), por lo que éstos factores no deben ser olvidados para dicho proceso.

En cuanto a las características psicológicas, la bibliografia las divide en aquellas necesarias para adquirir la condición de experto y aquellas para manifestar dicha condición (Baker \& Horton, 2004). Con respecto a las primeras, se establece que un alto nivel de motivación es esencial para alcanzar el éxito (Singer \& Orbach, 1999). Y con respecto a las segundas, se considera que los expertos deben manejar los estados de ansiedad (Gould, Petlichkoff, Simons \& Vevera, 1987), mantener la concentración y evitar los errores (Gould, Dieffenbach \& Moffett, 2002), como variables más importantes.

Atendiendo a las características sociales, se debe destacar a los padres como principales agentes socializadores a estas edades. La influencia de los padres variará a lo largo del proceso de desarrollo, pasando de ser los guías o directores, hasta llegar a consolidarse como una forma de apoyo más general. Es importante que los padres apoyen a sus hijos emocionalmente, facilitando las demandas psicológicas que requiere el entrenamiento; y económicamente, permitiéndoles el acceso a mejores entrenadores, equipamientos y medios de entrenamiento, ya que está demostrado que esto permite acumular mayor nivel de práctica y de mayor calidad (Baker, et al., 2003).

Otras variables culturales que influyen en el proceso de formación del deportista son (Baker, et al., 2003):

1. La importancia cultural. El arraigo que el deporte puede tener en la sociedad repercute en la televisión y en que los deportistas se consideren héroes nacionales. Lo cual implica que el deporte se convierta en parte de la identidad nacional, destinándose por ello parte del dinero público 
a generar grandes sistemas de clubes donde se empieza a practicar desde muy pequeño, con el apoyo del sistema educativo.

2. El clima predominante y la presencia de unas instalaciones determinadas. Ambos factores pueden favorecer la práctica de un deporte establecido y la ausencia de práctica de otro, así como la posibilidad de práctica durante determinados periodos de tiempo más propicios para un deporte que otro.

\section{Conclusión}

El objetivo fundamental de este trabajo de revisión ha sido analizar los factores principales que caracterizan a los expertos en el ámbito del deporte. Con este fin se han dividido dichos factores en genéticos, de entrenamiento, y psico-sociales. Sin embargo, reunir unas características genéticas excelentes combinado con un entrenamiento adecuado, desarrollado en un contexto ideal no garantiza el éxito. La complejidad de las relaciones entre la inmensidad de variables que afectan al desarrollo de la condición de experto hacen incierto el proceso. Por lo que a la hora de establecer conclusiones con respecto a un deporte específico hay que ser muy preciso teniendo presentes estas limitaciones.

A partir de esta realidad y siendo conscientes de que a edades muy tempranas, la dificultad para optimizar el entrenamiento y el rendimiento parece residir en el hecho de no estar definido el desarrollo final del sujeto, tanto físico como psíquico. Se pueden dar unas pautas que orienten el trabajo de entrenamiento en las primeras etapas $(9-11$ años), para los deportes colectivos, sin especificar las necesidades de cada disciplina, puesto que no es el objetivo de este trabajo. Para ello se centra la atención en lo que la bibliografía determina como constatado a la hora de proponer un trabajo de entrenamiento. Por lo que se elabora una propuesta basada en ocho aspectos fundamentales para el entrenamiento y la formación deportiva integral:

1. Desarrollar un trabajo general para generar una base, principalmente, basada en las habilidades motrices básicas y el desarrollo físico global, que corresponden con la edad.

2. Trabajar los contenidos propios bajo situaciones de toma de decisión, que permitan el desarrollo de la percepción y la anticipación, de la capacidad de elección y de ejecución. Para ello es necesario proponer tareas en las que los sujetos asuman responsabilidades ante las posibilidades que se planteen y no sólo someterlos a meras repeticiones para la corrección técnica.

3. Asegurar el mayor tiempo de práctica a todos los sujetos, teniendo en cuenta las características individuales. Dentro del tiempo de práctica, posibilitar la práctica activa en contacto con el móvil o balón, puesto este tipo de participación resulta muy significativa en el individuo.

4. En relación al tercer aspecto, adaptar la práctica a las necesidades y habilidades de los sujetos, con el fin de posibilitar un desarrollo integral. La adaptación se debe realizar, principalmente, sobre las reglas o normas, los equipamientos y las tareas.

5. Tras la adquisición de una base inicial de habilidades, variar las condiciones de práctica para asentar con más riqueza las destrezas iniciales. Esto permitirá aumentar el bagaje de experiencias de los sujetos y por tanto aumentará su capacidad de toma de decisión y de ejecución.

6. Utilizar tareas analíticas, que mejoran la calidad de la ejecución y permite corregir; tareas globales, que desarrollan las capacidades que se van a dar en el juego y en especial la toma de decisión; y el juego o la propia competición, que por sí mismo constituyen un medio de aprendizaje. Los diferentes tipos de tareas se deben realizar en función de las necesidades de los individuos y los objetivos.

7. Trabajar la motivación del jugador remarcando, principalmente, aspectos como la capacidad de lucha, el espíritu de superación, la inteligencia, la capacidad para transformar la agresividad en competencia y entrenamiento, la capacidad de emprendimiento, el ánimo ante las derrotas y la capacidad para no sobrevalorar la victoria.

8. Involucrar a los padres en el proceso de formación de sus hijos. Aportarles información sobre su función e importancia en el proceso, para que en ningún caso su falta de formación conlleve a errores.

\section{Futuras líneas de investigación}

Tras el análisis de la bibliografía revisada, se han suscitado una serie de dudas o problemas que pueden dar lugar a la realización de nuevas investigaciones. Algunas de estas ideas son:

1. Fruto de las conclusiones extraídas y atendiendo a la cantidad y diversidad de variables que influyen en el desarrollo del experto, se requiere la realización de estudios multidisciplinares que combinen conocimientos biológicos, psicológicos y sociológicos. Así, por ejemplo, son necesarios estudios que exploren la relación entre personalidad y condición de experto, para que desde la psicología se tengan argumentos para trabajar con los deportistas. Igualmente, es necesario ahondar en el conocimiento sobre la relación entrenamiento y predisposición genética, sobre la cual se encuentran pocos datos.

2. En función de la nueva visión del proceso de formación de expertos, se estima oportuno el desarrollo de estudios que muestren los planteamientos seguidos en dicho proceso, a la vez que se analiza su validez. En este mismo sentido, sería conveniente analizar si los planteamientos de entrenamiento son eficaces para el progreso del experto, durante las primeras y últimas etapas del desarrollo de los individuos.

3. En la mayoría de trabajos en los que se relaciona la cantidad de práctica deliberada y el desarrollo del rendimiento, el análisis de la cuantificación de la cantidad de práctica se hace mediante una técnica retrospectiva. Esto supone una posible limitación en cuanto a la fiabilidad de los datos, por lo que se requiere la realización de estudios que lleven un control riguroso de dicho parámetro.

4. Puesto que se sabe que el entrenamiento y en concreto la práctica condicionan en un alto porcentaje la condición de experto, son necesarias nuevas investigaciones que permitan asegurar que la práctica es la variable más importante que caracteriza al deportista experto.

5. Al igual que existen diferencias entre los deportes individuales y colectivos, en cuanto a importancia que se le da a diferentes aspectos del entrenamiento, se debe analizar si los resultados de estudios que se realizan en áreas más específicas y/o en los deportes individuales son aplicables a los deportes colectivos. Con el fin de mostrar cómo de aplicable son los datos que se poseen para el deporte en general.

6. En relación a las variables de edad biológica y cronológica, sería adecuado analizar si la influencia es tan determinante para todos los deportes como se apunta en diferentes trabajos. $\mathrm{Y}$ en este sentido, la distribución cronológica de las categorías deportivas debería someterse a estudio, con el sentido de generar categorías más competitivas y por tanto, que contribuyesen en mayormedida al desarrollo de los individuos.

7. Son necesarios nuevos trabajos que se centren en analizar las características por las cuales se definen a los expertos en deportes específicos, es decir, sólo centrándose en un solo deporte. De esta manera se contribuirá en la orientación de las propuestas de trabajo de forma mucho más concisa y acertada, al centrar la atención en los aspectos que realmente son determinantes en una determinada disciplina deportiva. Igualmente son imprescindibles trabajos que señalen con precisión las diferencias adoptadas entre la concepción de talento y de experto en los diferentes deportes.

\section{Bibliografía}

Abernethy, B. (1990). Anticipation in squash: differences in advance cue utilization between experts and non-expert players. Journal of Sports Sciences, 8, 17-34.

Baker, J. \& Côté, J. (2003). Resources and commitment as critical factors in the development of 'gifted' athletes. High Ability Studies, 14, 139-140.

Baker, J., Côté, J. \& Abernethy, B. (2003). Learning from the experts: practice activities of expert decision-makers in sport. Research Quarterly for Exercise and Sport, 74, 342-347.

Baker, J., Côté, J., \& Deakin, J. (2005). Cognitive characteristics of expert, middle of the pack, and back of the pack ultra-endurance triathletes. Psychology of Sport and Exercise, 6(1), 551-558. 
Baker, J. \& Horton, S. (2004). A review of primary and secondary influences on sport expertise. High Ability Studies, 15(2), 211-228.

Baker, J., Horton, S., Robertson-Wilson, J., \& Wall, M. (2003). Nurturing sport expertise: factors influencing the development of elite athletes. Journal of Sports Science and Medicine, 2, 1-9.

Baxter-Jones, A. \& Helms, P. (1994). Born too late to win? Nature, 370, 186.

Blázquez, D. (1995). Iniciación deportiva y deporte escolar. Barcelona: Inde.

Boyce, B. A., Coker, C. A. \& Bunker, L. K. (2006). Implications for variability of practice from pedagogy and motor learning perspectives: finding a common ground. Quest, 58, 330-343.

Cárdenas, D. (2003). El proceso de formación táctica colectiva desde una perspectiva constructivista. En A. López, C. Jiménez \& R. Aguado (Eds.), Didáctica del baloncesto en las etapas de formación (pp. 179-209). Madrid: Editores.

Chalip, L. \& Green, B. C. (1998). Establishing and maintaining a modified youth sport program: lessons from hotelling's location game. Sociology of Sport Journal, 15, 326-342.

Chi, M. T. H., Feltovich, P. J. \& Glaser, R. (1981). Categorization and representation of physics problems by experts and novices. Cognitive Science, 5, 121-152.

Côté, J. (1999). The influence of the family in the development of talent in sports. The Sports Psychologist, 13, 395-417.

Côté, J., Macdonald, D. J., Baker, J. \& Abernethy, B. (2006). When «where» is more important than «when»: birthplace and birthdate effects on the achievement of sporting expertise. Journal of Sports Sciences, 24(10), 1065-1073.

Committee on Sports Medicine and Fitness \& Committee on School Health (2001). Organized sports for children and preadolescents. Pediatrics, 107(6), 1459-1462.

Damas, J. S. \& Julián, J. A. (2002). La enseñanza del voleibol en las escuelas deportivas de iniciación. Madrid: Gymnos.

Del Villar, F. \& Iglesias, D. (2003). La enseñanza de la toma de decisiones en el deporte escolar. Conferencia presentada en el I Congreso Europeo de Educación Física FIEP. Cáceres.

Del Villar, F., Julián, J. A., Iglesias, D. \& Fuentes, J. P. (2003). El papel de los contenidos procedimentales en la adquisición del conocimiento en elárea de Educación Física. Retos. Nuevas tendencias en Educación Física, Deporte y Recreación, 6, 38-44.

Dodds, P., Griffin, L. L., \& Placek, J. H. (2001). A selected review of the literature on development of learners' domain-specific knowledge. Journal of Teaching in Physical Education, 20, 301-313.

Dyson, B., Griffin, L. L. \& Hastie, P. (2004). Sport education, tactical games, and cooperative learning: theoretical and pedagogical considerations. Quest, 56, 226-240.

Edwards, S. (1994). Born too late to win? Nature, 370, 186.

Ericsson, K. A., Krampe, R. T. \& Tesch-Römer, C. (1993). The role of deliberate practice in the acquisition of expert performance. Psychological Review, 100, 363-406.

Ericsson, K. A. \& Lehmann, A. C. (1996). Expert and exceptional performance: evidence of maximal adaptation to task constraints. Annual Review of Psychology, 47, 273-305.

French, K. E. \& Thomas, J. R. (1987). The relation of knowledge development to children's basketball performance. Journal of Sport Psychology, 9, 15-32.

Giménez, F. J. \& Sáenz-López, P. (2000). Aspectos teóricos y prácticos de la iniciación al baloncesto. Huelva: Diputación de Huelva.

Ginsburg, K. R., Committee on Communications \& Committee on Psychosocial Aspects of Child and Family Health. (2007). The importance of play in promoting healthy child development and maintaining strong parent-child bonds. Pediatrics, 119(1), 182-191

Gould, D., Dieffenbach, K. \& Moffett, A. (2002). Psychological characteristics and their development in Olympic champions. Journal of Applied Sport Psychology, 14, 172-204.

Gould, D., Petlichkoff, L., Simons, J. \& Vevera, M. (1987). Relationship between competitive state anxiety inventory-2 scores and pistol shooting performance. Journal of Sport Psychology, 9, 33-42.

Green, K. \& Magill, R. A. (1995). Variability of practice and contextual interference in motor skill learning. Journal of Motor Behavior, 27(4), 299-309.
Hamilton, B. (2000). East African running dominance: what is behind it? British Journal of Sports Medicine, 34, 391-394.

Helsen, W. F., Hodges, N. J., Van Winckel, J. \& Starkes, J. L. (2000). The roles of talent, physical precocity and practice in the development of soccer expertise. Journal of Sport Sciences, 18, 727-736.

Hernández, J., Castro, U., Cruz, H. Gil, G., Guerra, G, Quiroga, M., et al. (2000). La iniciación a los deportes desde su estructura y dinámica. Barcelona: Inde.

Hynes, J. M. (2002). Practice and motor learning. Physical Educator, $59(2), 58-62$.

Landin, D. L. \& Herbert, E. P. (1997). A comparison of three practice schedules along the contextual interference continuum. Research Quarterly for Exercise and Sport, 68, 357-361.

Lee,A. M. (1996). How the field evolved. In S. J. Silverman \& C. D. Ennis (Eds.), Student learning in physical education. Applying research to enhance instruction (pp. 9-34). Champaign: Human Kinetics.

Lorenzo, A. (2003). Estudio del pensamiento de los entrenadores sobre el proceso de detección de talentos en baloncesto. Revista Motricidad. European Journal of Human Movement, 10, 23-51.

Nielsen, T. M., \& McPherson, S. L. (2001). Response selection and execution skills of professionals and novices during singles tennis competition. Perceptual and Motor Skills, 93, 541-555.

Pierón, M. (1989). Didáctica de las actividades físicas y deportivas. Madrid: Gymnos.

Piñar, M. I. (2005). Incidencia del cambio de un conjunto de reglas de juego sobre algunas de las variables que determinan el proceso de formación de los jugadores de minibasket (9-11 años). Granada: Universidad de Granada.

Piñar, M. I. \& Cárdenas, D. (2004). El minibasket 3x3 y sus aportaciones al juego del niño durante la competición. En A. López, C. Jiménez \& C. López (Eds.), II Curso de Didáctica del baloncesto en las etapas de formación (pp. 16-37). Madrid: Editores.

Rankinen, T., Pérusse, L., Rauramaa, R., Rivera, M. A., Wolfarth, B. \& Bouchard, C. (2001). The human gene map for performance and health-related fitness phenotypes. Medicine \& Science in Sports and Exercise, 33, 855-867.

Rankinen, T., Pérusse, L., Rauramaa, R., Rivera, M. A., Wolfarth, B. \& Bouchard, C. (2002). The human gene map for performance and health-related fitness phenotypes: the 2001 update. Medicine \& Science in Sports and Exercise, 34, 1219-1233.

Regimbal, C., Deller, J. \& Plimpton, C. (1992). Basketball size as related to children's preference, rated skill and scoring. Perceptual and Motor Skills, 75, 867-872.

Rovegno, I., Nevett, M. \& Babiarz, M. (2001). Chapter 5. Learning and teaching invasion-game tactics in 4 th grade: introduction and theoretical perspective. Journal of Teaching in Physical Education 20 341-351

Ruiz, L. M. \& Sánchez, F. (1997). Rendimiento Deportivo. Claves para la optimización de los aprendizajes. Madrid: Gymnos.

Ruiz, L. M., Sánchez, M., Durán, J. \& Jiménez, C. (2006). Los expertos en el deporte: su estudio y análisis desde una perspectiva psicológica. Anales de Psicología del Deporte, 22(1), 132-142.

Schmidt, R. A. (1975). A schema theory of discrete motor skill learning. Psychological Review, 82, 225-260.

Silva, F. M., Fernandes, L. \& Celani, F. O. (2001). Desporto de crianças e jovens - um estudo sobre as idades de iniciaçao. Revista Portuguesa de Ciencias do Desporto, 1(2), 45-55.

Silverman, S., Devillier, R. \& Ramirez, T. (1991). The validity of academy learning time-physical education (ALT-PE) as a process measure of achievement. Research Quarterly for Exercise and Sport, 62(3), 319-325.

Singer, R. N. \& Orbach, I. (1999). Persistence, excellence, and fulfilment. In R. Lidor \& M. Bar-Eli (Eds.), Sport psychology: linking theory and practice (pp. 167-191). Morgantown: Bookcrafters.

Smith, R. E. \& Christensen, D. S. (1995). Psychological skills as predictors of performance and survival in professional baseball. Journal of Sport \& Exercise Psychology, 17, 399-415.

Solanellas, F. (1999). Detección de talentos en tenis. Revista de Psicología del Deporte, 8(2), 259-270.

Williams, A. M. \& Hodges, N. J. (2005). Practice, instruction and skill acquisition in soccer: challenging tradition. Journal of Sports Sciences, 23(6), 637-650. 